Анищенко А. C. Кухарь В. В.

Присяжный А. Г.

\title{
АППРОКСИМАЦИЯ КОНТУРА БОКОВОЙ ПОВЕРХНОСТИ ЗАГОТОВОК ПРИ ОСАДКЕ ПЛОСКИМИ БОЙКАМИ
}

Осадка плоскими бойками формирует в заготовке бочкообразную боковую поверхность. Расчет энергосиловых параметров осадки предполагает наличие формул, достоверно описывающих контур этой поверхности.

Для описания контура боковой поверхности осаженной заготовки используют несколько видов формул (табл. 1), аппроксимирующих численные значения, полученные по результатам натурных экспериментов [1-3]. Главное отличие формул состоит в величине радиуса кривизны образующей контура, который может быть как постоянным (для окружности), так и изменяющимся в сторону увеличения или уменьшения относительно радиуса окружности.

Вид формулы выбирают до аппроксимации экспериментальных значений, что является методологической ошибкой, поскольку именно набор экспериментальных точек, а не субъективное мнение исследователя определяет вид формулы. Такое может быть в случае, если формула аппроксимации имеет универсальный вид, а все возможные варианты изменения радиуса кривизны боковой поверхности определяются коэффициентами аппроксимации, входящими в формулу. Погрешность аппроксимации зависит как от вида формулы, так и от числа и точности измерений экспериментальных данных. При неточном аналитическом описании контура боковой поверхности изменяется расчетный объем заготовки относительно исходного, снижается точность расчетов давления и силы осадки.

Таблица 1

Некоторые формулы аппроксимации поверхности бочкообразного образца после осадки

\begin{tabular}{|c|c|}
\hline Формула & Обозначения \\
\hline$y=\frac{2\left(D_{m}-D_{б}\right)}{H^{2}} x^{2}+\frac{D_{б}}{2}$ & $D_{\sigma}, D_{m}, H$ - диаметры бочки торца и высота заготовки \\
\hline$\frac{x^{2}}{a^{2}}+\frac{y^{2}}{b^{2}}=1$ & $a=\frac{H}{2 \sqrt{1-\left(\frac{D_{m}}{D_{\sigma}}\right)^{2}}}, \quad b=\frac{D_{6}}{2}$ \\
\hline $\begin{array}{c}\text { окружность } \\
x^{2}+(y-b)^{2}=R^{2}\end{array}$ & $b=\frac{D_{\sigma}^{2}-D_{m}^{2}-H^{2}}{4\left(D_{\sigma}-D_{m}\right)} \quad R=b=\frac{\left(D_{\sigma}-D_{m}\right)^{2}+H^{2}}{4\left(D_{\sigma}-D_{m}\right)}$ \\
\hline $\begin{array}{c}\text { косинус } \\
y=\operatorname{acos}(b x)\end{array}$ & $a=\frac{D_{\sigma}}{2} \quad b=\frac{2}{H} \arccos \left(\frac{D_{m}}{D_{\sigma}}\right)$ \\
\hline $\begin{array}{l}\text { косинус гиперболический } \\
y=\operatorname{acosh}(b x)\end{array}$ & $a=\frac{D_{m}}{2} \quad b=\frac{2}{H} \operatorname{argcos}\left(\frac{D_{6}}{D_{m}}\right)$ \\
\hline
\end{tabular}


В литературе имеются сведения [4-6] об использовании суперэллипса Габриэля Ляме в качестве универсальной формулы для описания контуров деформируемых заготовок. Суперэллипс в зависимости от значений входящих в него коэффициентов может описывать замкнутые фигуры типа параллелограмма, астроиды, двух парабол, симметричных относительно оси х, окружности, эллипса и сквиркла с различными радиусами скругления вершин.

Целью настоящей работы является совершенствование методики аппроксимации контура боковой поверхности осаживаемой заготовки с использованием суперэллипса Ляме.

Суперэллипс Ляме в общем виде записывается формулой:

$$
(x / a)^{n}+(y / b)^{m}=1,
$$

где $n$ и $m$ - показатели степени; $a, b$ - радиусы (полуоси) суперэллипса.

Чаще всего используется вариант суперэллипса, в котором $n=m=N[6]$.

Графики, описываемые формулой (1), симметричны относительно координатных осей, поэтому достаточно рассмотреть их поведение в первом квадранте. На рис. 1 представлена схема выбора координат аппроксимирующих функций относительно геометрических размеров осаженной бочкообразной заготовки.

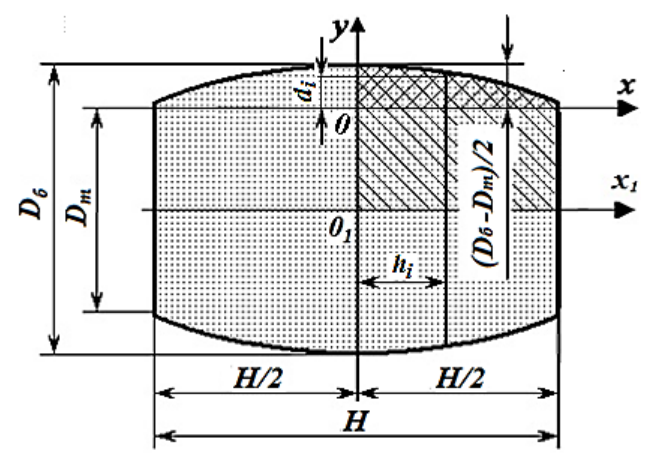

Рис. 1. Схема к выбору системы координат аппроксимирующих функций

Поскольку для более точной аппроксимации суперэллипсом экспериментальные точки контура должны быть определены во всем диапазона $\left[0, x_{\max }=a\right]$, то выбираем систему размерных или безразмерных координат $y 0 x$ с пределами измерений по обеим осям от нуля до значений $a$ и $b$. Ось $x$ параллельна оси образца и совпадает с образующей цилиндра с диаметром, равным диаметру торца $D_{m}$. Ось $y$ пересекает ось $x$ в плоскости максимального диаметра бочки образца $D_{\sigma}$. При необходимости безразмерность координат $X_{i}$ и $Y_{i}$ обеспечиваем соответственно делением измеренного реального размера $h_{i}($ мм) на максимально возможный размер, который равняется половине высоты осаженной заготовки $H / 2$, то есть $\left[X_{i}=h_{i} /(H / 2)\right]$, а также делением измеренного реального размера:

$$
d_{i}=\left(D_{\sigma}^{i}-D_{m}\right) / 2
$$

при $x=h_{i}$ на максимально возможный размер, который равняется полуразности диаметров бочки и торца заготовки, то есть:

$$
Y_{i}=\frac{\left(D_{\sigma}^{i}-D_{m}\right) / 2}{\left(D_{\sigma}^{\max }-D_{m}\right) / 2}
$$

Бочкообразный контур заготовок изучали по результатам осадки двух партий свинцовых заготовок. Первая партия имела исходные размеры Ø20 × 20 мм, была осажена со степенью деформации $\varepsilon=\left(H_{0}-H\right) / H_{0}=0,4$ в режиме сухого трения и с применением смазок. Определенный по методике Губкина коэффициент трения составлял $\mu=0,29 \ldots 0,41$. Вторая 
партия заготовок имела исходные размеры $\varnothing 30 \times 60$ мм и была осажена в режиме сухого трения со степенями деформации $\varepsilon=0,1 \ldots 0,5$ и шагом $\Delta \varepsilon=0,1$. На боковой поверхности заготовок выполняли кольцевые риски, отстоящие друг от друга на расстоянии, равном 0,1 от исходной высоты $H_{0}$. Искривление контура боковой поверхности изучали по результатам изменения диаметра этих рисок при осадке.

Результаты осадки первой партии заготовок представлены на рис. 2. Геометрическая форма кривых 1-6 показывает, что даже при осадке с минимальным коэффициентом трения (смазки «Укринол-7» и ОГВ-75) контур боковой поверхности заготовок не является частью сферы. С увеличением коэффициента трения (кривые 4,5) увеличивается разность между максимальным диаметром бочки и диаметром торца, а боковой контур заготовок еще больше отклоняется от сферообразного.

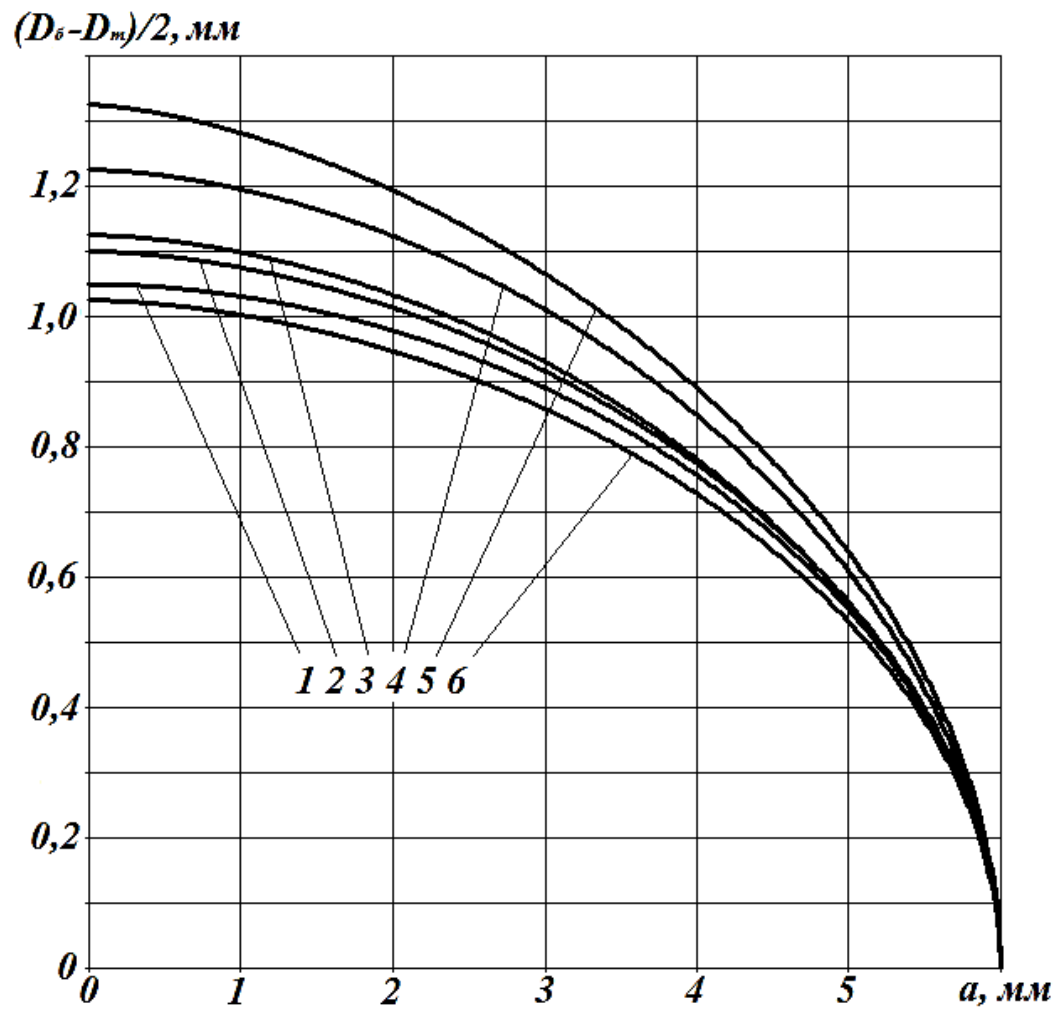

Рис. 2. Контуры заготовок с $H_{0} / D_{0}=1$ после осадки с $\varepsilon=0,4$ и $\mu$ :

$1-0,29 ; 2-0,32 ; 3-0,34 ; 4-0,35 ; 5-0,41 ; 6-0,30$

В табл. 2 представлены коэффициенты, полученные при аппроксимации графиков на рис. 2 суперэллипсом (1), в котором коэффициент $a=6$ был равен половине высоты осаженной заготовки $(12 / 2=6$ мм). Значения коэффициентов $b$ определяли по формуле (2).

Таблица 2

Значения коэффициентов суперэллипса для графиков на рис. 2

\begin{tabular}{|c|c|c|c|c|c|c|c|c|c|}
\hline № графика & $b$ & $n$ & $m$ & $N$ & № графика & $b$ & $n$ & $m$ & $N$ \\
\hline 1 & 1,05 & 1,88 & 1,92 & 1,90 & 4 & 1,225 & 1,73 & 1,87 & 1,80 \\
\hline 2 & 1,1 & 1,75 & 1,93 & 1,83 & 5 & 1,325 & 1,55 & 1,92 & 1,72 \\
\hline 3 & 1,125 & 1,75 & 1,86 & 1,80 & 6 & 1,025 & 1,76 & 1,97 & 1,86 \\
\hline
\end{tabular}

Коэффициенты $n$ и $m$ (в первую очередь $-n$ ) позволяют на качественном уровне определить отклонение контура боковой поверхности осаженной заготовки от сферообразного. Чем больше положительное значение величины $j=2-n(2>n>1)$, тем больше контур 
похож на параболу. С ростом отрицательного значения $j(2,5>n>2)$ контур приобретает эллипсоидную форму. В нашем случае бочкообразование было наиболее интенсивным при осадке без смазки $(b=1,325)$ : боковой контур заготовки максимально отличался от сферообразного и величина $j_{\max }=2-1,55=0,45$.

На рис. 3 представлена эволюция контура боковой поверхности, а в табл. 3 - параметры ее аппроксимации при осадке второй партии заготовок.

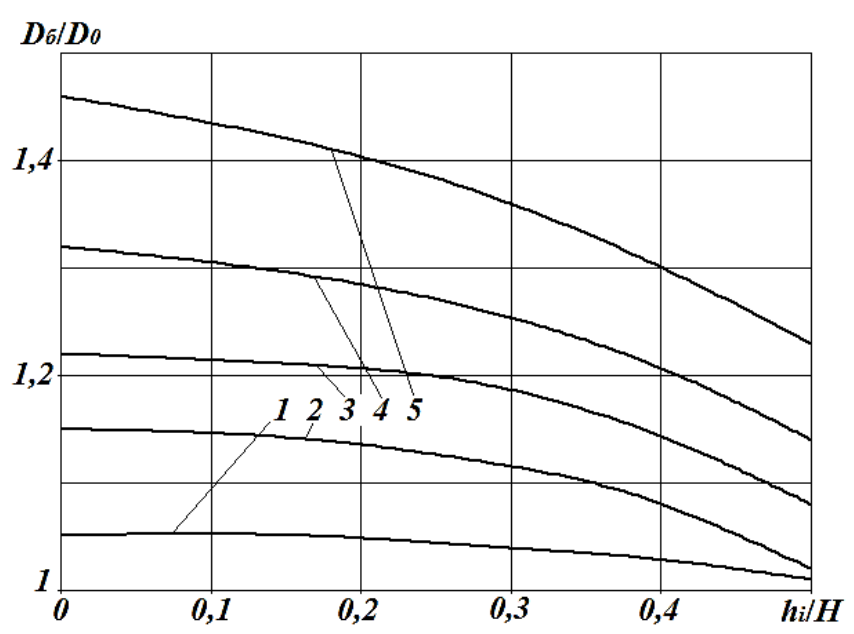

$\mathrm{a}$

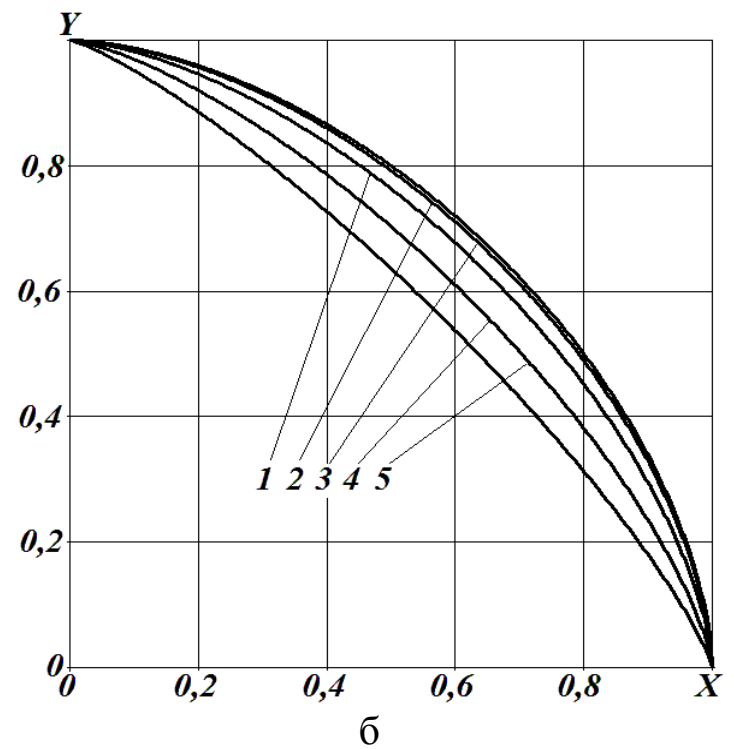

Рис. 3. Распределение в координатах $y 0_{l} x_{1}$ (a) и $y 0 x$ (б) относительного диаметра бочки вдоль оси заготовки, осаженной со степенью деформации $\varepsilon$ :

$$
1-0,1 ; 2-0,2 ; 3-0.3 ; 4-0,4 ; 5-0,5
$$

Относительно монотонный характер уменьшения коэффициента $n$ с ростом степени деформации при осадке указывает, что в интервале $\varepsilon=0,1 \ldots 0,5$ с ростом $\varepsilon$ контур бочки все более отклоняется от окружности в сторону параболы. Аномалии изменения $n$ при $\varepsilon=0,1 \ldots 0,2$, вероятно, связаны с незначительным двойным бочкообразованием, которое может иметь место, поскольку относительная исходная высота заготовок $H_{0} / D_{0}=2$ достаточно большая.

Таблица 3

Значения коэффициентов суперэллипса для графиков на рис. 3, б

\begin{tabular}{|c|c|c|c|c|c|c|c|}
\hline № графика & $n$ & $m$ & $N$ & № графика & $n$ & $m$ & $N$ \\
\hline 1 & 2,02 & 0,78 & 1,55 & 4 & 1,56 & 0,8 & 1,38 \\
\hline 2 & 2,15 & 1,44 & 1,65 & 5 & 1,47 & 0,92 & 1,23 \\
\hline 3 & 1,75 & 1,63 & 1,69 &
\end{tabular}

Аппроксимацию бочкообразных контуров суперэллипсом, кроме методов статистического анализа, можно осуществить, исходя из равенства объемов заготовки до и после деформации. Допустим, исходная заготовка объемом $V_{0}=\pi D_{0}^{2} H / 4$ после осадки имеет бочкообразный контур, который можно описать уравнением (1), и объем $V_{б}$, определяемый по формуле:

$$
V_{\text {б }}=\pi \int_{0}^{\frac{\mathrm{H}}{2}} y^{2} d x .
$$


Интеграл от функции (1) в явном виде не существует, поэтому функцию (1) разлагаем в ряд Тейлора по $(x-1)$ и интегрируем первые два члена этого ряда. Для упрощения интегрирования формулы (4) заменим систему координат $y 0 x$ на $y 0_{l} x_{l}$ (рис. 1). Кроме того, чтобы избежать замены $y$ на $y+\left(D_{m} / 2\right)$ и усложнения интегрирования, заменяем коэффициенты $a$ и $b$ на $A$ и $B$ при неизменности аппроксимированных значений $n$ и $m$ :

$$
(x / A)^{n}+(y / B)^{m}=1 .
$$

После совмещения координатной оси $x$ с осью $x_{1}$ :

$$
\begin{aligned}
& \text { при } x=0 \quad y=B=\left(D_{m} / 2\right)+b=D_{\sigma} / 2 ; \\
& \text { при } x=a=H / 2 A=\frac{H}{2}\left[1-\left(\frac{D_{m} / 2}{D_{\sigma} / 2}\right)^{m}\right]^{1 / n} .
\end{aligned}
$$

Два первых члена ряда Тейлора для суперэллипса (1) равны:

$$
\begin{gathered}
y(1)=B A^{-n / m}\left(A^{n}-1\right)^{1 / m} \\
y^{\prime}(1)=-\frac{n}{m} B A^{-n / m}\left(A^{n}-1\right)^{\frac{1}{m}-1}
\end{gathered}
$$

и ряд Тейлора имеет вид:

$$
y=B A^{-n / m}\left(A^{n}-1\right)^{\frac{1}{m}}\left[1-\frac{n}{m} \frac{x-1}{A^{n}-1}\right]
$$

Обозначив: $z=B^{2} A^{-2 n / m}\left(A^{n}-1\right)^{\frac{2}{m}}, w=\frac{n}{m}\left(A^{n}-1\right)^{-1}$, после интегрирования (4) получим:

$$
V_{\text {б }}=\pi z\left[\frac{H}{2}(1+w)^{2}+\frac{w H^{2}}{2}(w-1)+\frac{w^{2} H^{3}}{24}\right] .
$$

Условие постоянства объема заготовки до и после деформации $V_{\sigma}=V_{0}$ примет вид:

$$
D_{0}^{2} H_{0}=2 z H\left[(1+w)^{2}+w H(w-1)+\frac{w^{2} H^{2}}{12}\right] .
$$

По разности значений обеих частей уравнения (5) можно судить о погрешности аппроксимации суперэллипсом контура заготовки при осадке плоскими бойками.

Замеры координат большого числа экспериментальных точек на боковой поверхности осаженных заготовок обусловливают высокий уровень систематической и случайной погрешности измерений и трудоемкость работ. Уравнение (5) позволяет упростить процесс аппроксимации. Если принять $n=m=N$, то для определения коэффициента аппроксимации $N$ из формулы (5) достаточно произвести замеры только параметров $D_{\sigma}, D_{m}$ и $H$. При необходимости определения $n$ и $m$ следует дополнительно осадить с той же степенью деформации заготовку с другими размерами, но с тем же соотношением $H_{0}^{\prime} / D_{0}^{\prime}$ и измерить полученные значения $D_{\sigma}^{\prime}, D_{m}^{\prime}$ и $H^{\prime}$. В результате образуется система двух уравнений вида (5), из которой рассчитываются значения $n$ и $m$. Достоверность расчетов гарантируется соблюдением условия постоянства объемов $V_{0}=V_{\sigma}$. 
В табл. 2-3 с погрешностью 4-12 \% представлены значения коэффициента $N$ аппроксимации контуров при осадке обеих партий заготовок. Поскольку $N<2$, можно утверждать, что бочкообразный контур осаженных заготовок имеет форму параболы.

\section{ВЫВОДЫ}

Суперэллипс Ляме является универсальной формулой для аппроксимации любого возможного вида контура боковой поверхности осаженных заготовок. Вид контура может быть определен по величине коэффициентов, входящих в суперэллипс.

Приведена формула, позволяющая по величине диаметров бочки, торца и высоте осаженной заготовки определить суперэллипс аппроксимации.

\section{СПИСОК ИСПОЛЬЗОВАННОЙ ЛИТЕРАТУРЫ}

1. Tonneau (formules). Available at: https://fr.wikipedia.org/wiki/Tonneau_(formules).

2. Torrentea G. Numerical and experimental studies of compression-tested copper: proposal for a new friction correction. Materials Research. 2018; 21(4). DOI: http://dx.doi.org/10.1590/1980-5373-MR-2017-0905

3. Aluko O., Adeyemi M.B. Warm Compression Tests of Aluminum Alloy. Journal of Materials Engineering and Performance. 1998, 7(4), pp. 474-478.

4. Sadowski A.J., Michael Rotter J. Exploration of novel geometric imperfection forms in buckling failures of thin-walled metal silos under eccentric discharge. International Journal of Solids and Structures. 2013, 50(5), 781-794. DOI: http://doi:10.1016/j.ijsolstr.2012.11.017

5. Anishchenko A., Kukhar V., Artiukh V., Arkhipova O. Application of G. Lame's and J. Gielis' formulas for description of shells superplastic forming. MATEC Web of Conferences, $060072018 . \quad$ DOI: https://doi.org/10.1051/matecconf /201823906007

6. Overfelt P.L. Superspheroid geometries for radome analysis. Naval air warfare center weapons division china lake, CA 93555-6001, Sept. 1994, 40 p. Available at: https://apps.dtic.mil/dtic/tr/fulltext/u2/a285405.pdf

\section{REFERENCES}

1. Tonneau (formules). Available at: https://fr.wikipedia.org/wiki/Tonneau_(formules).

2. Torrentea G. Numerical and experimental studies of compression-tested copper: proposal for a new friction correction. Materials Research. 2018; 21(4). DOI: http://dx.doi.org/10.1590/1980-5373-MR-2017-0905

3. Aluko O., Adeyemi M.B. Warm Compression Tests of Aluminum Alloy. Journal of Materials Engineering and Performance. 1998, 7(4), pp. 474-478.

4. Sadowski A.J., Michael Rotter J. . Exploration of novel geometric imperfection forms in buckling failures of thin-walled metal silos under eccentric discharge. International Journal of Solids and Structures. 2013, 50(5), 781-794. DOI: http://doi:10.1016/j.ijsolstr.2012.11.017

5. Anishchenko A., Kukhar V., Artiukh V., Arkhipova O. Application of G. Lame's and J. Gielis' formulas for description of shells superplastic forming. MATEC Web of Conferences, $060072018 . \quad$ DOI: https://doi.org/10.1051/matecconf /201823906007

6. Overfelt P.L. Superspheroid geometries for radome analysis. Naval air warfare center weapons division china lake, CA 93555-6001, Sept. 1994, 40 p. Available at: https://apps.dtic.mil/dtic/tr/fulltext/u2/a285405.pdf

Анищенко А. С. - канд. техн. наук, ст. науч. сотр., доц. каф. ОМД ПГТУ; E-mail: aas540628@gmail.com

Кухарь В. В. $\quad$ - д-р техн. наук, проф., зав. каф. ОМД ПГТУ;

E-mail: kvv_mariupol@gmail.com

Присяжный А. Г. - канд. техн. наук, доц. каф. ОМД ПГТУ;

E-mail: agp87514@gmail.com

ПГТУ - Приазовский государственный технический университет, г. Мариуполь. 